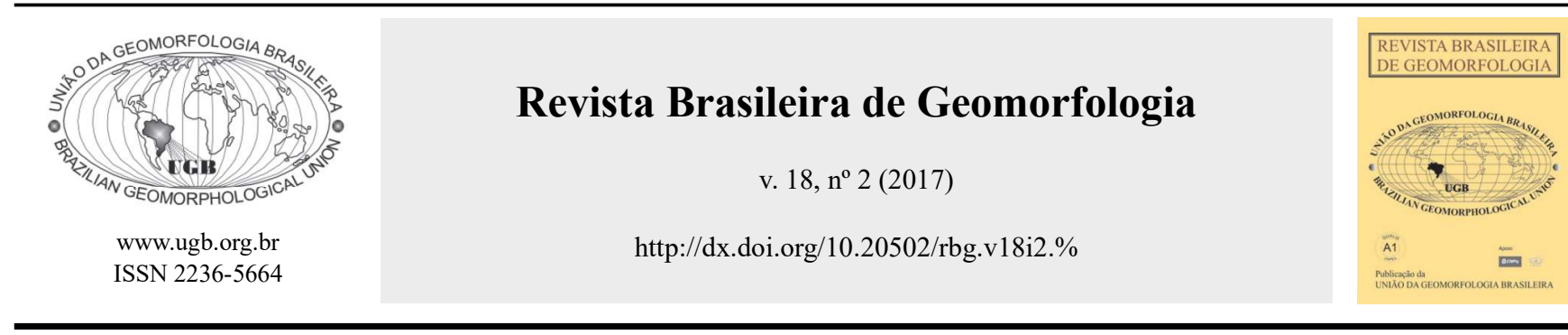

\title{
ROCHAS DE PRAIA "BEACHROCKS" DA ILHA DO CABO FRIO - LITORAL DO ESTADO DO RIO DE JANEIRO - SUDESTE BRASILEIRO: GÊNESE E GEOCRONOLOGIA
}

\section{THE CABO FRIO ISLAND BEACHROCKS - RIO DE JANEIRO STATE COAST SHORE - BRAZILIAN SOUTHEAST: GENESIS AND GEOCHRONOLOGY}

\author{
Julia Varella Malta \\ Museu Nacional, Universidade Federal do Rio de Janeiro \\ Quinta da Boa Vista, Rio de Janeiro, Rio de Janeiro. CEP: 20940-040. Brasil
}

Email:jvmalta@ymail.com

João Wagner Alencar Castro

Museu Nacional, Universidade Federal do Rio de Janeiro Quinta da Boa Vista, Rio de Janeiro, Rio de Janeiro. CEP: 20940-040. Brasil Email:jwacastro@gmail.com

Camila Areias de Oliveira

Museu Nacional, Universidade Federal do Rio de Janeiro Quinta da Boa Vista, Rio de Janeiro, Rio de Janeiro. CEP: 20940-040. Brasil Email:milareias@gmail.com

Claudio Couto Reis

Universidade Petrobrás

Rua Ulysses Guimarães, 565, Rio de Janeiro, Rio de Janeiro. CEP: 20211-150. Brasil

Email: claudio.couto@petrobras.com.br

\section{Informações sobre o Artigo}

Recebido (Received):

25/01/2017

Aceito (Accepted):

$25 / 04 / 2017$

\section{Palavras-chave:}

Rochas de Praia; Variação do Nível Relativo do Mar; Análise Petrográfica.

\section{Keywords:}

Beachrocks; Relative SeaLevel Fluctuation; Petrography Analyses.

\section{Resumo:}

Rochas de praia (beachrocks) marcam a linha de costa pretérita e a constituição sedimentar de paleopraias. Em ambiente de micro-marés definem com precisão a posição do nível médio do mar na ocasião do processo de litificação. O presente trabalho objetiva estudar os "beachrocks" da Ilha do Cabo Frio, Arraial do Cabo - Rio de Janeiro do ponto de vista geológico, geomorfológico, petrográfico e geocronológico. O método de trabalho constou de coleta de material, análise petrográfica e datação ao radiocarbono. Resultados obtidos sugerem que os "beachrocks" da referida ilha foram formados entre 11.900 a 3.800 cal anos A.P. Essas idades correspondem a condições de nível do mar abaixo do atual, no nível atual e acima do nível marinho em relação ao atual durante o Holoceno. Identificou-se através deste trabalho a passagem entre o Pleistoceno e o Holoceno no Estado do Rio de Janeiro, corroborando propostas de outros autores. 


\begin{abstract}
:
Beachrocks are rocks that indicate a former shoreline as well as the sedimentary constitution of ancient beaches. In micro-tidal environments, they accurately determine the position of the medium sea level during the lithification process. They are formed by the beach sediment cemented within the intertidal zone. The objective of this paper is to study study the beachrocks of the Cabo Frio Island, Arraial do Cabo - Rio de Janeiro from the geological, geomorphological, petrographic and geochronological point of view. The methods applied in this study consisted in sampling, petrographic analysis and radiocarbon dating. The results suggest that the beachrocks of this island were formed between 11.900 and 3800 . These ages correspond to sea level conditions below the current level, at the current level and above the sea level in relation to the current level during the Holocene. The transition between the Pleistocene and the Holocene in the State of Rio de Janeiro was identified by this paper, corroborating the proposals of other authors.
\end{abstract}

\section{Introdução}

O termo "beachrock" no significado amplo tem sido aplicado a um variado conjunto de materiais litificados na zona litorânea, incluindo aqueles com cimentos de ferro e sílica. Aplica-se exclusivamente aos sedimentos praiais cimentados por carbonato de cálcio, na zona de inter-marés, limitados às baixas latitudes (Hopley, 1986). O processo de litificação dos "beachrocks" é mais comum em locais de temperaturas mais elevadas, caracterizado pelo alto índice de evaporação da água do mar (Friedman e Sanders, 1978; Cooper, 1991; Friedman et al., 1992). Porém também podem ser encontrados em locais de clima temperado a frio (Cooray, 1968; Chivas et al.,1986; Khalaf, 1988; Holail e Rashed, 1992; Ertek et al., 2008; Erginal et al., 2008).

Os "beachrocks", são utilizados amplamente como indicadores de variações do nível relativo do mar (VNRM) por Alexandersson (1972), Dalongeville e Sanlaville (1984a, 1984b), Goudie (1969), Guilcher (1961), Scholten (1971), Stoddart e Cann (1965), Martin, et al (1997), Castro et al (2014). Por isso essas rochas vêm sendo estudadas por diversos autores, entre estes, Roncarati $e$ Neves (1976), Martin et al. (1984), Suguio e Tessler (1984), Flexor et al. (1984), Dias e Silva (1984), Suguio e Martin (1987), Villwock (1994), Turcq et al. (1999) e Suguio (1999, 2003a e 2003b) e Castro et al (2014). Em geral essas rochas marcam a linha de costa pretérita e a constituição sedimentar de paleopraias. Porém existem controvérsias quanto ao papel das rochas de praia "beachrocks" como indicador de variações do nível relativo do mar, por exemplo Kelletat (2006) que afirma que os "beachrocks" não representam indicadores de VNRM representativos. Em quanto Ramsey (1995) e Castro et al (2014) apontam como excelentes indicadores abióticos de VRNR na projeção vertical em relação ao zero atual.

Os primeiros relatos sobre "beachrocks" datam de
1817 por Sir Francis Beauford conforme (Goudie, 1969). Seguidos por Von Buch (1825), Moresby (1835), Darwin (1841), Dana (1849) e Branner (1904). Desde então afloramentos de "beachrock" foram identificados em muitas áreas costeiras localizadas em diversas partes do mundo, Ginsburg (1953) na Florida, Cooray (1968) no Sri Lanka, Chivas et al. (1986) na Australia, Meyers (1987) no Hawaii, Khalaf (1988) no Golfo Pérsico, Amieux et al. (1989) na África, Holail e Rashed (1992) no Mediterrâneo e no Mar Vermelho, Kindler e Bain (1993) na América Central, Ertek et al. (2008) em Chipre, Erginal et al. (2008) na Turquia e Thomas (2009) na Índia.

No Brasil estudos sobre os "beachrocks" são registrados desde Darwin (1841) e Branner (1904). No entanto a maioria dos trabalhos concentra-se no litoral do nordeste brasileiro, destacando Van Andel e Laborel (1964), Mabesoone (1964), Morais (1967), Bigarella (1975), Caldas et al. (2006), Bezerra et al. (1998 e 2005), Guerra et al. (2005), Ferreira Jr. (2005), Vieira e Ros (2006), Vieira et al. (2007). No litoral do estado do Rio de Janeiro registram-se os trabalhos de Macedo (1963), Muehe (1984), Muehe e Ignarra (1987), Castro et al (2011), Silva et al (2014), Castro et al.(2014).

\section{Gênese dos "Beachrocks"}

A formação dos "beachrocks" pode ser dividida em três categorias, uma orgânica e duas inorgânicas, uma a partir da água doce e outra a partir da água do mar. A categoria orgânica provém da ação direta ou indiretade organismos microbiológicos, da decomposição da matéria orgânica e da atividade algálica como processos responsáveis pela precipitação de cimentos em "beachrocks" (Webb et al., 1999; Neumeier, 1999, Vieira, 2005). A primeira categoria inorgânica provém da mistura de águas subterrâneas e meteóricas com a água marinha. Russel (1959 e 1962) ao pesquisar "beachrocks" em ilhas 
do Caribe afirma que a cimentação primária ocorre ao longo do nível freático na forma de cutículas de calcita. A segunda categoria inorgânica leva em consideração a ação apenas da água do mar. Baseia-se na identificação da aragonita como cimento primário, inicialmente sob a forma de cristais aciculares ou fibrosos. Segundo Ferreira Jr. (2010), neste caso, a precipitação do cimento aragonítico ocorre em profundidade rasa ou na face de praia, após processos de evaporação e aquecimento da água do mar. Hanor (1978) propôs que a perda de $\mathrm{CO}_{2}$ a partir de águas praiais saturadas em carbonato seja o processo responsável pela precipitação dos cimentos em "beachrocks".

Nos "beachrocks" pode-se encontrar desde material inteiramente vulcânico (Tietz e Muller,1971) a grãos inteiramente carbonáticos (Kindler e Bain, 1993), assim como, quartzo e fragmentos de rochas (Chaves e Sial, 1998). Em contraste com a cimentação lenta de muitos ambientes diagenéticos, a formação das rochas de praia é excepcionalmente rápida e ocorre em algumas dezenas de anos, fato esse evidenciado pela ocorrência de inúmeros objetos modernos cimentados nestas rochas (Holail e Rashed, 1992). De modo geral apresentam-se paralelo a linha de costa com ligeiro declive em relação a plataforma continental. Essas características geomorfológicas são identificada nos afloramentos através da estratificação cruzada de baixo angulo (Malta et al, 2015)

\section{3. Área de Estudo}

A ilha de Cabo Frio localiza-se no segmento leste do estado do Rio de Janeiro, no município de Arraial do Cabo. A morfologia é caracterizada por uma feição alongada, ocupando uma área de aproximadamente 6,5 $\mathrm{km}^{2}$. Os dois pontos mais elevados alcançam uma altitude de aproximadamente $390 \mathrm{~m}$ e $260 \mathrm{~m}$ em relação ao nível do mar atual (Figura 1).
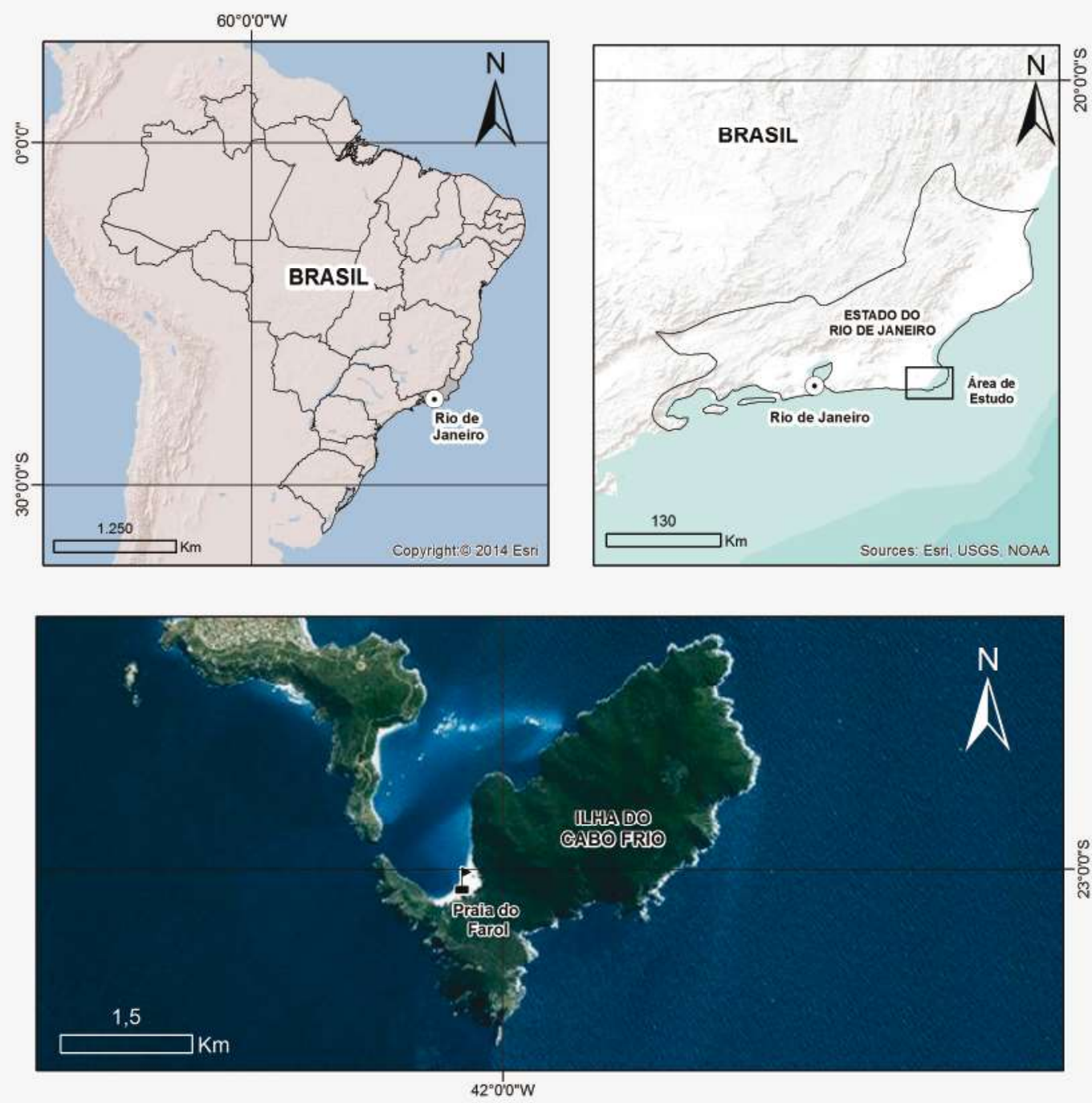

Figura 1 - Localização da área de estudo no contexto do litoral do Estado do Rio de Janeiro. 
Nesse segmento ocorrem dois domínios geológicos - geomorfológicos distintos: 1) rochas de embasamento e 2) cobertura sedimentar Cenozóica. As rochas de embasamento do Paleoproterozóico caracterizam-se por promontórios (headland) constituídos por ortognaisses félsicos, ortoanfibolitos e metassedimentos intercalados com anfibolitos. Ocorrem também intrusões alcalinas do Mesozóico-Cenozóico (Schmitt, 2001). A cobertura Cenozóica caracteriza-se por sedimentos de origem continental (Formação Barreiras) e por sedimentos costeiros / marinhos constituídos por deltas, praias arenosas, cordões litorâneos, dunas obliquas, lagoas, lagunas e pântanos (Castro et al, 2014). Estudos de variações relativas do nível do mar de Castro et al (2014) identificaram antigas posições de linha de praia referente à última transgressão holocênica no litoral do Estado do Rio de Janeiro. O máximo transgressivo ocorreu por volta de 4590 - 5100 cal anos A.P. O nível marinho encontrava-se a 3,0 m acima do atual, resultando na formação de uma grande paleolaguna (Castro et al, 2014).

Nessa região, sistemas de ilhas barreiras e cordões litorâneos se estabeleceram frontalmente à paleolaguna durante o máximo transgressivo a 5.100 anos A.P (Castro et al., 2009). À medida que o nível do mar foi baixando o ambiente lagunar foi se transformado em áreas de pântanos (Martin et al., 1997). A ocorrência de terraços marinhos constituídos por areias a cascalhos, provenientes do intemperismo dos promontórios (headland) associam-se a esse evento máximo transgressivo do Holoceno (Turq et al. 1999). A plataforma continental interna da região é recoberta por areia de granulometria média a fina e por uma estreita faixa de lama que se estende até a profundidade de 25 m (Silva et al, 2011).

O clima de ondas é predominantemente de bom tempo e do quadrante nordeste. A ocorrência de marulhos (swell) provenientes de Anticiclones do Atlântico Sul, associa-se as frentes frias polares, com direção leste-sudeste (Silva, 2009). A área de estudo insere-se na costa central do estado do Rio de Janeiro, dominada por micro maré. O regime de maré é do tipo assimétrico e semi-diurno com preamares na ordem de $1,0 \mathrm{~m}$, baixa-mares entre 0.06 e $0.025 \mathrm{~m}$ em relação ao nível de redução de 0.67 $\mathrm{m}$ definido pela Marinha do Brasil (Silva, 2009). Nessa região ocorre um fenômeno oceanográfico conhecido como ressurgência costeira. Esse fenômeno é gerado por ventos de nordeste, produzidos por um centro atmosférico de alta pressão semi-permanente sobre o Oceano Atlântico Sul, permitindo a ascensão de águas frias, ricas em nutrientes. As anomalias de temperatura negativa da superfície do mar causadas pelo direcionamento dos ventos na ressurgência costeira estão presentes na plataforma continental do sudeste brasileiro, especialmente durante o verão (Gyllencreutz et al, 2012). Quando os ventos de nordeste persistem por vários dias, uma forte ressurgência tende a ocorrer com a queda da temperatura superficial do mar para até $15^{\circ} \mathrm{C}$ próximo da costa de Cabo Frio. Estas temperaturas são cerca de $10^{\circ} \mathrm{C}$ mais frias em relação às áreas adjacentes na costa do sudeste brasileiro (Fonseca, 2012).

\section{Materiais e método}

O método de trabalho consistiu de coleta de amostras emersas e submersas de "beachrocks". As amostras submersas foram obtidas através de mergulho autônomo. A posição geográfica dos afloramentos foi definida através de um rastreador geodésico DGPS Pro Mark 2.0. Durante essa etapa de trabalho, utilizou-se à carta 1503, $6^{\mathrm{a}}$ edição, datum horizontal Córrego Alegre, enseada do Cabo Frio, escala 1:20.000 (Marinha do Brasil) e informações obtidas em campo através de um ecobatímetro acoplado ao DGPS.

Foram coletadas um total de 10 amostras de material nas profundidades de $-4,5$ e - 3,0 m e 10 amostras emersas no nível médio do mar atual e $1,5 \mathrm{~m}$ em relação ao corrente nível, perfazendo um total de 20 amostras. Em cada afloramento identificou-se as estruturas sedimentares e observações macroscópicas. A coleta do material submerso foi realizada por meio de mergulho autônomo, utilizando cilindros de ar comprimido. Os afloramentos emersos foram fotografados através de uma câmera digital Canon Rebel T3 e os submersos através de uma Go Pro Hero 3.

Foram selecionadas 15 amostras para a confecção de lâminas delgadas, levando em consideração a representatividade do fragmento coletado e o grau de intemperismo. Foi realizado um tratamento por impregnação com resina epoxy azul através de técnicas padronizadas de identificação da mineralogia dos cimentos carbonáticos conforme metodologia adotada por Warne, (1962) e Friedman (1971). Tal procedimento visou determinar aspectos texturais básicos, identificação dos componentes detríticos, individualização de feições diagenéticas e reconhecimento de uma sequência de ocorrência das mesmas. Esta etapa da pesquisa foi realizada no Laboratório de Laminação do Departamento de Geologia e 
Paleontologia do Museu Nacional e no Laboratório de Laminação do Departamento de Geologia ambos pertencentes à UFRJ.

Na análise petrográfica utilizou-se um microscópio Carl Zeiss ${ }^{\circledR}$ Axioskop 40. A análise foi realizada em luz transmitida onde se procedeu a identificação mineralógica, de microestruturas sedimentares e identificação dos bioclastos presentes. Foram contados 300 pontos para determinar o tipo de rocha. Verificou-se que as rochas com mais de $50 \%$ de material siliciclástico foram consideradas rochas siliciclástica e nas rochas constituídas por mais de $50 \%$ de material carbonático foram consideradas nesta análise como carbonáticas com base nos trabalhos de Terra (2010). Em seguida 5 amostras do material coletado em diferentes níveis batimétricos e altimétricos foram encaminhadas para datação ao radiocarbono ${ }^{14} \mathrm{C}$ em rocha total. O procedimento de datação foi realizado pelo Laboratório Beta Analytic Inc, Miami - Estados Unidos através do método Radiometric Plus. Esse método utiliza parcelas maiores das amostras para realizar as análises. As amostras foram submetidas a um pré-tratamento em laboratório, chamado de condicionamento ácido.

\section{Resultados}

As rochas de praia "beachrocks" da Ilha do Cabo Frio estudada, encontram-se em 4 níveis distintos, sendo 2 emersos nas posições $0,0 \mathrm{~m}$ (nível do mar atual) e $1,5 \mathrm{~m}$ acima deste, - 3,0 e - 4,5 $\mathrm{m}$ abaixo do nível do mar atual (Figura 2). Do ponto de vista geomorfológico caracterizam-se por corpos alongados de pequena extensão e largura com ligeiro caimento em direção a face litorânea e /ou zona de surf. Apresentam arcabouço geralmente idêntico aos sedimentos de praia sobrejacente ou adjacente.

\subsection{Petrografia dos “Beachrocks” da Ilha do Cabo Frio}

Macroscopicamente as rochas dos 4 afloramentos são constituídas por arenitos médios, formados principalmente por bioclastos, com a presença de vermetídeos e cracas em alguns locais. Petrograficamente as amostras emersas apresentam características semelhantes. Os afloramentos submersos também se assemelham entre si. As rochas emersas podem ser classificadas como grainstones com partículas de 0,3 a 2,0 mm, formadas principalmente por fragmentos de rodófitas e bivalves, e cristaloclastos constituídos por quartzo, feldspato, fragmentos de rochas sedimentares e ígneas alcalinas (Figura $3 \mathrm{~A}$ e B). Apresentam-se moderadamente bem selecionadas com clastos subangulosos a arredondados e circularidade de moderada a muito alta. Constituem-se principalmentepor grãos flutuantes. São encontrados também contatos pontuais, alongados e côncavo convexos entre os bioclastos (Figura $3 \mathrm{C}$ ). Não foi identificada matriz no material analisado. Verificou-se a presença de cimento carbonático marinho, composta por franja prismática de calcita magnesiana (Figura $3 \mathrm{C}, \mathrm{D}, \mathrm{E}$ e F). Os cristaloclastos de feldspato são formados por cristais de microclima e plagioclásio com diferentes graus de alteração. Apresentam também bordas e clivagens parcialmente substituídas por carbonato e dissolvidas.

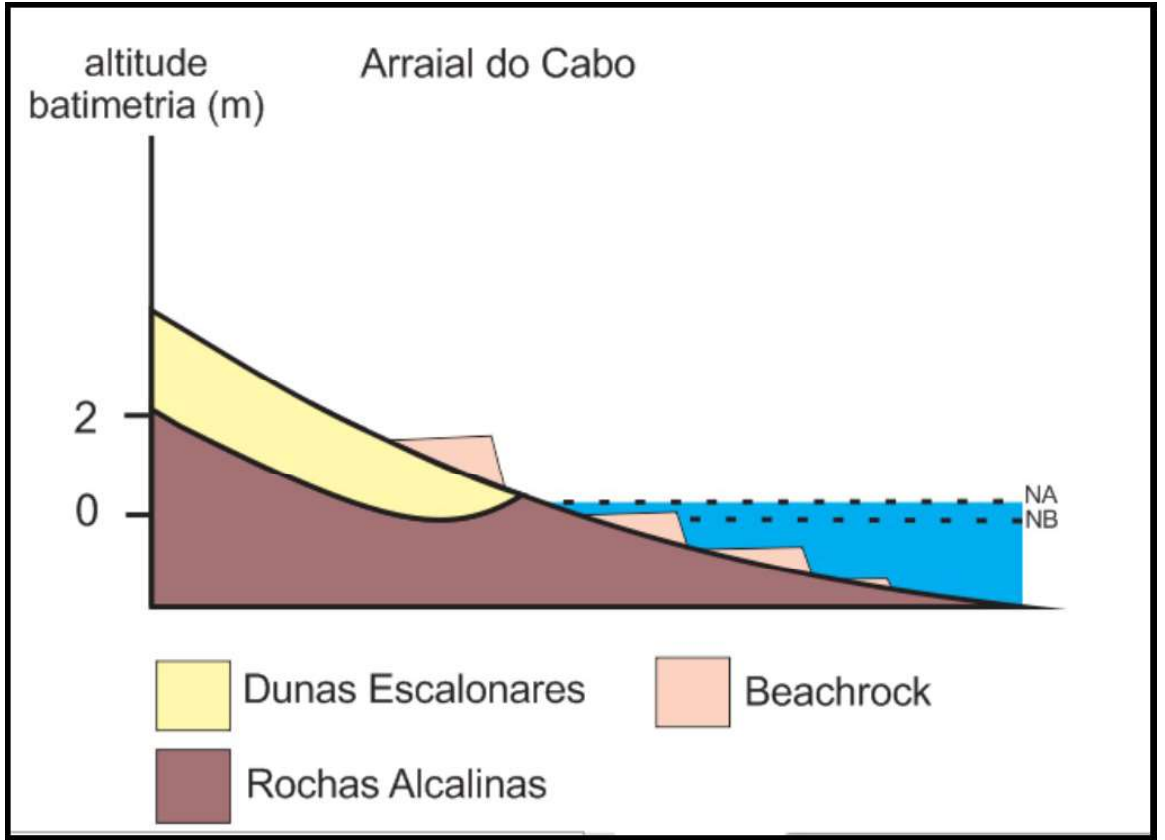

Figura 2 - Seção esquemática dos afloramento de "beachrocks" na ilha do Cabo Frio, Arraial do Cabo - Rio de Janeiro. 
Malta J. V. et al.

Foram identificadas grandes concentrações de bioclastos, entre 40 e $60 \%$, principalmente fragmentos de rodófitas. São encontrados também fragmentos de bivalves em até $10 \%$, representando estrutura lamelar cruzada com preservação dos grãos de quartzo
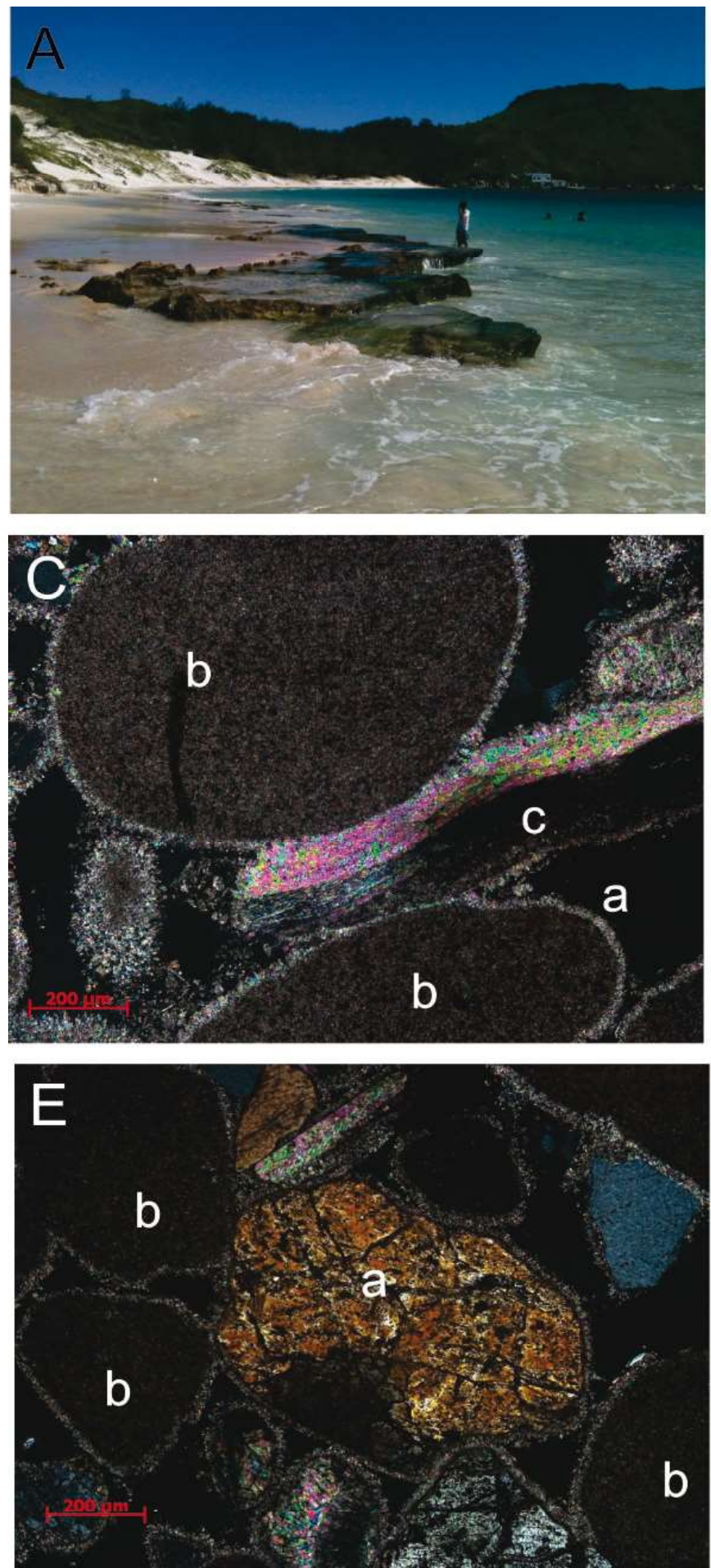

entre 10 e $15 \%$, feldspato até $5 \%$ e fragmentos de rocha até $10 \%$. Esse material é proveniente, tanto do embasamento como de origem sedimentar. Também são encontrados alguns grãos de hornblenda (Figura 3 E e F).
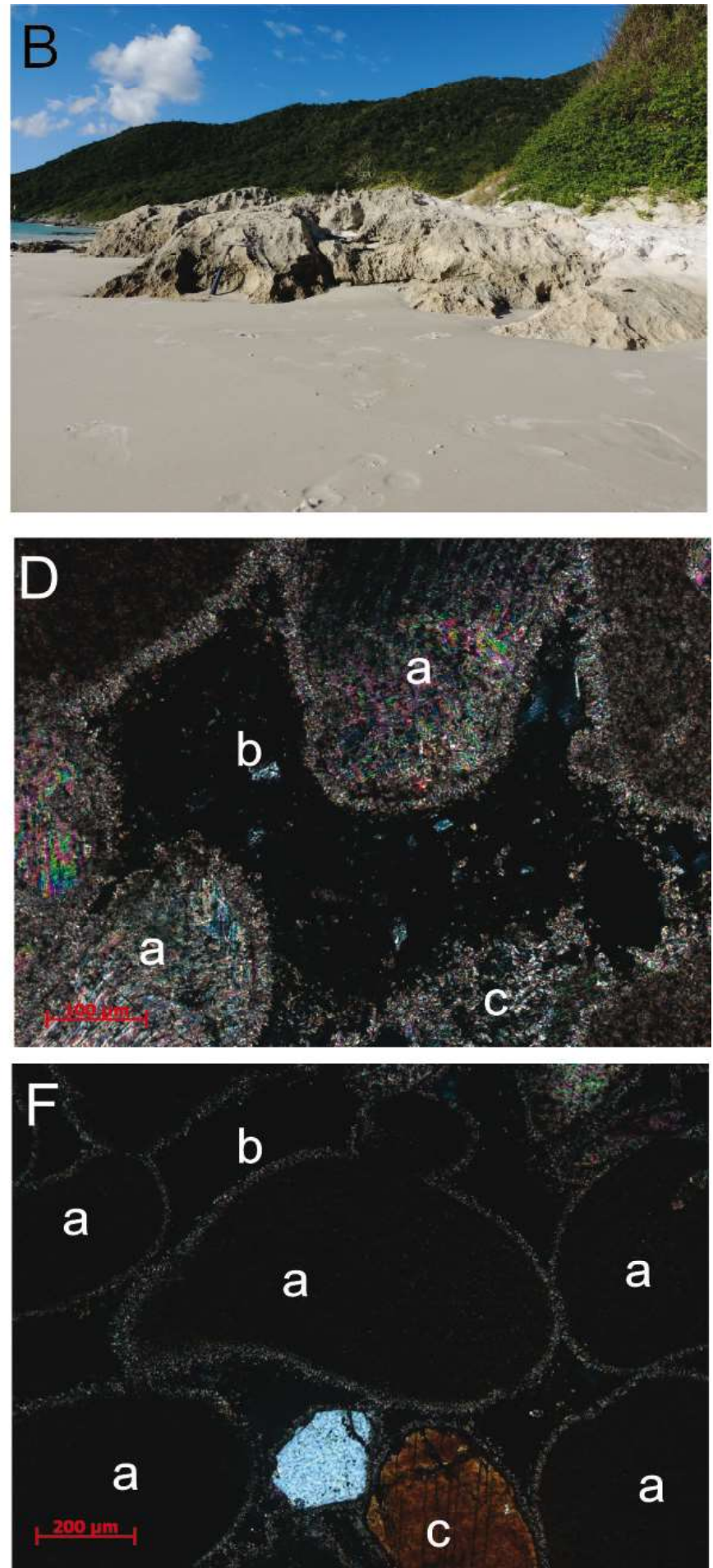

Figura 3 - (A) Afloramento de "beachrock" no nivel relativo do mar atual. (B) Afloramento de "beachrock" emerso com altitude 1,5m. (C) a Cimento do tipo franja prismática de calcita magnesiana. b. Fragmento de rodófita. $c$. Fragmento de bivalve com estrutura lamelar cruzada preservada. (D) a. Grão de feldspato alterado. b. Fragmento de rocha sedimentar deformado. (E) a. Grão de hornblenda. b. Fragmento de rodófitas. $(F)$ a. Fragmento de rodófitas. b. Cimento do tipo franja prismática de calcita magnesiana. c. Grão de hornblenda. 
As rochas submersas também podem ser classificadas como grainstones (Figura 4 A e B). Apresentam maior quantidade de bioclastos em relação às emersas. Caracterizam-se por grãos de 0,3 a $2,0 \mathrm{~mm}$, principalmente formados por rodófitas, fragmentos de bivalves em aproximadamente $90 \%$ (Figura $4 \mathrm{C}$ e D), cristaloclastos formados quartzo - feldspato, fragmentos de rochas sedimentares e ígneas alcalinas em até $10 \%$ (Figura 4 F). Rocha sem orientação preferencial, moderadamente bem selecionada com clastos subangulosos a arredondados e circularidade moderada a alta. Apresenta principalmente cristais flutuantes com contatos pontuais e alongados e até côncavo convexos, constituída por rocha altamente porosa entre 10 e $15 \%$ sem matriz.
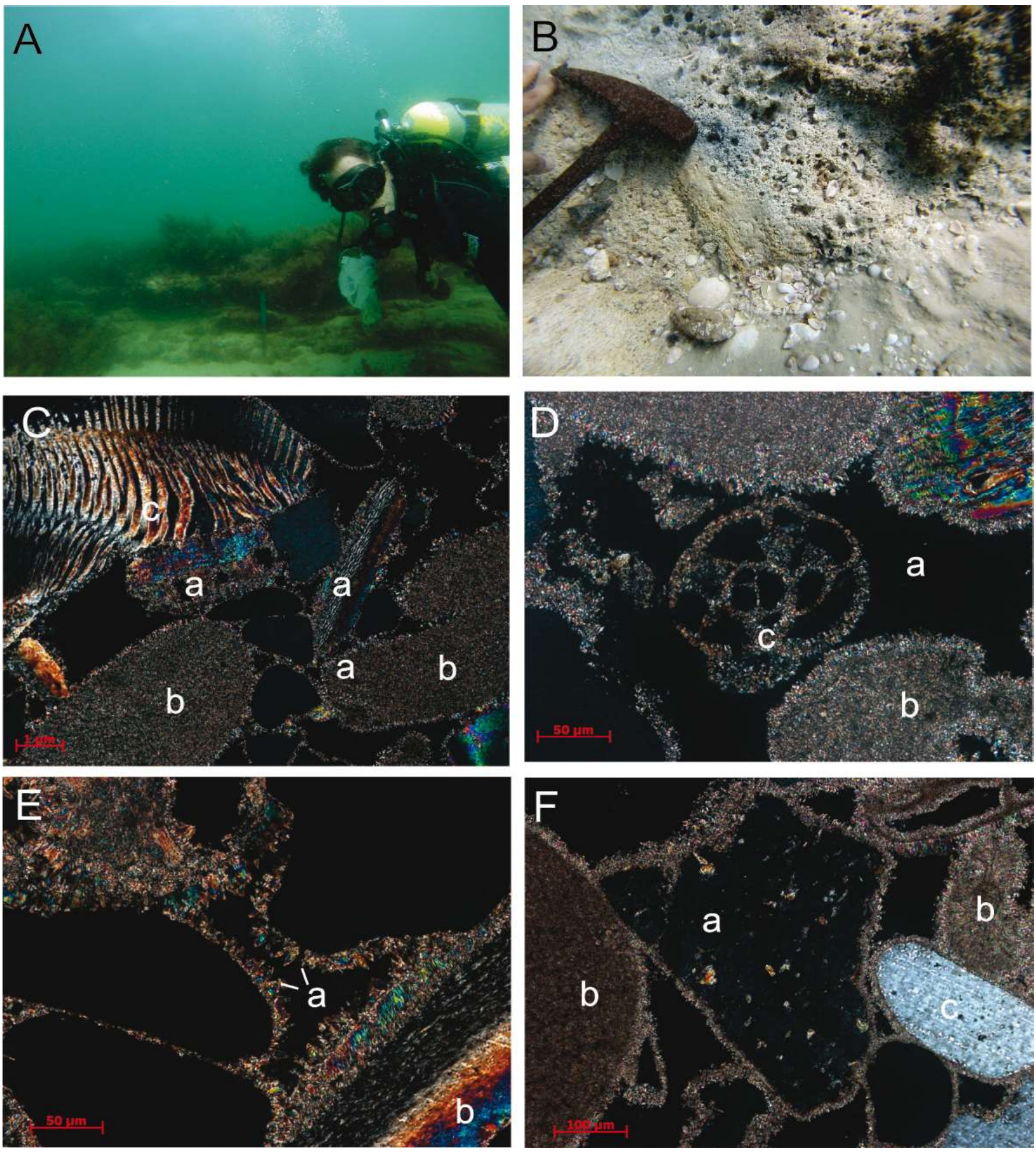

Figura 4 - (A) "Beachrock" submerso com batimetria de - 3,0 m. (B) "Beachrock” submerso com batimetria de - 4,5m. (C) a. Fragmento de gastrópode com estrutura zebra striping preservada. b. Fragmento de rodófita. (D) a. Poro. b. Fragmento de rodófita c. Fragmento de gastrópode. (E) a. Cimento do tipo franja prismática de calcita magnesiana. b. Fragmento de concha de bivalve com estrutura cruzada lamelar preservada. (F) a. Fragmento de rocha sedimentar. b. Fragmento de rodófita. c. Grão de quartzo. 
Apresenta cimentação carbonática marinha composta por franja prismática de calcita magnesiana mais proeminente que as rochas emersas (Figura $4 \mathrm{E}$ ). São encontradas grandes concentrações de bioclastos, principalmente rodófitas entre 60 e 70\% (Figura 4 C). Identificou-se também fragmentos de bivalves entre 10 e 15\% das amostras (Figura 4 C). Os cristaloclastos são formados principalmente por grãos de quartzo entre 10 e $15 \%$, feldspatos entre 5 e $10 \%$ e fragmentos de rocha até $10 \%$ das amostras de proveniente do embasamento cristalino alcalino e também de origem sedimentar (Figura 4 F). Também foram encontrados alguns cristaloclastos de hornblenda.
A idade ao radiocarbono ${ }^{14} \mathrm{C}$ da amostra Beta 248.721 , coletada na profundidade de $-4,5 \mathrm{~m}$, foi de $10.460+/-70$ anos A.P., calibrada em $2 \sigma$ entre 11.940 - 11.240 cal anos A.P. Na amostra Beta 243.863, profundidade de $-3,0 \mathrm{~m}$, foi de $10.250+/-50$, calibrada em $2 \sigma$ entre $11.149-11.337 \mathrm{cal}$ anos A.P. Na amostra Beta 248.722, nível médio do mar atual foi obtida uma idade de 7.090 +/- 60 A.P., calibrada em $2 \sigma$ entre 7.910 - 7.690 cal anos A.P. Na amostra Beta 248.723, 1,5 m acima do nível médio do mar atual foi obtida idade de 3.210 +/- 80 anos A.P., calibrada em $2 \sigma$ entre 3.132 - 2.860 cal anos A.P (Tabela 1).

Tabela 1: Datação ao radiocarbono ${ }^{14} \mathrm{C}$ de 4 amostras de "beachrocks" coletadas na ilha do Cabo Frio, Rio de Janeiro.

\begin{tabular}{ccccc}
\hline $\begin{array}{c}\text { Número da } \\
\text { amostra }\end{array}$ & Tipo de amostra & Altitude (m) & $\begin{array}{c}\text { Idade Calibrada } \\
\text { (anos cal. A.P. 2 })\end{array}$ & $\begin{array}{c}\text { Idade Convencional } \\
\text { (anos A.P.) }\end{array}$ \\
\hline $\begin{array}{c}\text { Beta Analytic } \\
248721\end{array}$ & Rocha total & $-4,5$ & $11940-11240$ & $10460 \pm 70$ \\
$\begin{array}{c}\text { Beta Analytic } \\
243863\end{array}$ & Rocha total & $-3,0$ & $11940-11240$ & $10250 \pm 50$ \\
$\begin{array}{c}\text { Beta Analytic } \\
248722\end{array}$ & Rocha total & 0,0 & $7910-7690$ & $7090 \pm 60$ \\
$\begin{array}{c}\text { Beta Analytic } \\
248723\end{array}$ & Rocha total & $+1,5$ & $3130-2860$ & $3210 \pm 80$ \\
\hline
\end{tabular}

\section{Discussão}

Segundo Hanor (1978) registra-se a presença de três categorias de "beachrocks" quanto ao ambiente de litificação, uma orgânica e duas inorgânicas. Através do presente trabalho identificou-se somente a categoria inorgânica submetida a influência de água marinha. Nas amostras analisadas identificou-se a presença de calcita magnesiana como cimento primário, principalmente sob a forma de franja prismática. Identificou-se também em todas as amostras, tanto emersas como submersas, a ocorrência cimento microcristalino secundário. Hanor (1978) sugere que a perda de $\mathrm{CO}_{2}$ a partir de águas praiais saturadas em carbonato é o principal responsável pelo processo de precipitação dos cimentos em "beachrocks".

Devido o surgimento da ressurgência costeira por volta de 3.500 anos A.P. não se observa a partir desse evento registros de "beachrocks" no litoral do Estado do Rio de Janeiro (Castro et al, 2014). A temperatura da água atual é um inibidor do processo de cimentação em "beachrocks" ao longo desse trecho de litoral. A temperatura da água do mar durante o processo de litificação dessas rochas encontrava-se mais quente em relação a atual (Castro et al. 2014). Tal fato é justificado pela ocorrência de algas Rhodophytado gênero Spongites Kützing, $1847 \mathrm{em}$ todas as amostras de "beachrocks" coletadas. Esse gênero aponta ambiente deposicional em águas tropicais com temperaturas acima de $23^{\circ} \mathrm{C}$, desde a transição Pleistoceno - Holoceno até aproximadamente 3500 anos A.P. (Castro et al., 2012). Essas condições de paleotemperaturas da água acima de $23^{\circ} \mathrm{C}$ são muito diferentes dos registros atuais, cuja média, situa-se em torno de $18^{\circ} \mathrm{C}$ devido ao fenômeno da ressurgência costeira. O processo de litificação dos "beachrocks" nesse intervalo de tempo, ocorreu em condições de águas bem mais quentes em relação à atual. A zona de ressurgência do litoral de Arraial do Cabo - Brasil, durante esse intervalo de tempo, encontrava-se afastada em relação à posição atual (Castro, et al 2014).

Nos afloramentos de "beachrocks" da Ilha do Cabo Frio são identificadas grandes concentrações de bioclastos principalmente fragmentos de rodófitas em até $90 \%$ (Spotorno-Oliveira et al 2016). São encontrados também fragmentos de bivalves que apresentam estrutura lamelar cruzada preservada em até $10 \%$. Esses 
dados divergem dos resultados obtidos por Silva et al. (2014) em afloramentos de "beachrocks" das praias da Barra da Tijuca e Itaipuaçu no Estado do Rio de Janeiro. Nessas praias foram identificados grãos de quartzo entre 65 e $70 \%$, grãos de plagioclásio e feldspato entre 3 e $5 \%$ e bioclastos em percentuais pouco representativos entre 5 a $7 \%$ da composição da rocha. Castro et al. (2012) estudaram afloramentos de "beachrocks" da Ilha do Cabo Frio, descrevendo a cimentação carbonática constituída por calcita, no entanto nesse trabalho foi descrito como franja prismática de calcita magnesiana.

Os resultados aqui obtidos apresentados apontam para semelhanças, em termos de composição, estudos sobre os "beachrocks" em outras praias da costa do Estado do Rio de Janeiro e do nordeste do Brasil (Silva et al., 2014, Mansur et al., 2011, Vieira et al., 2005). No entanto a variedade de bioclasto é bastante diferente, ou seja, na área estudada, o percentual de bioclastos é de 40 a $90 \%$ constituído principalmente por rodophytas e bivalves. Enquanto que nos "beachrocks" do nordeste brasileiro registra-se a presença de gastrópodes, bivalves, foraminíferos, algas e equinoides vermelho (Vieira et al., 2005).

Registram-se também outras ocorrências de " $b e a$ chrocks" no litoral do Estado do Rio de Janeiro nas praias da Barra da Tijuca no município do Rio de Janeiro, praia de Itaipuaçu em Maricá (Silva et al., 2014) e na praia de Jaconé, no município de Saquarema (Mansur et al., 2011). Apresentam cimentação composta por franja prismática de calcita magnesiana, indicando condições de litificação similares às dos "beachrocks" da Ilha do Cabo Frio. Apesar dos afloramentos de "beachrocks" no nordeste brasileiro apresentarem em sua composição cimento composto por franja de calcita magnesiana prismática similares aos encontrados na Ilha do Cabo Frio, diferem dos últimos por apresentarem óxido de ferro no cimento proveniente da Formação Barreiras (Vieira et al., 2005).

\section{Conclusão}

Nos beachrocks estudados, identificou-se a presença de calcita magnesiana como cimento primário, principalmente sob a forma de franja prismática. Identificou-se também em todas as amostras, a ocorrência cimento microcristalino secundário. Através do presente trabalho, observou-se somente a categoria inorgânica, submetida a influência de água marinha, sugerindo ambiente deposicional marinho raso, provavelmente associado ao ambiente de praia (estirâncio) e ante-praia (zona de surf) submetido a ação de ondas e correntes de deriva litorânea.

Verificou-se nesse trabalho algumas semelhanças em termos de composição, petrografica e mineralógica do cimento dos "beachrocks" aqui estudados com outros afloramentos localizados na costa do nordeste brasileiro. A composição do cimento é praticamente a mesma, composta por franja de calcita magnesiana prismática. A principal diferença reside na presença de óxido de ferro no cimento proveniente da Formação Barreiras nos afloramentos do nordeste brasileiro. $\mathrm{Na}$ área estudada, o percentual de bioclastos é de 40 a 90\% constituído principalmente por rodophytas e bivalves. A fonte desse material é a plataforma continental interna caracterizada por águas mais quente em relação às condições atuais.

Do ponto de vista geomorfológico os "beachrocks" da Ilha do Cabo Frio, Rio de Janeiro, apresentam-se em quatro níveis altimétricos - batimétricos distintos, sendo dois submersos e dois emersos. Os afloramentos submersos marcam a transição Pleistoceno - Holoceno no litoral do Estado do Rio de Janeiro, registrada entre 11.940 a $11.149 \mathrm{cal}$ anos A.P. Os emersos, apresentam idade entre 7910 - $7690 \mathrm{cal}$ anos AP, correspondente ao nível do mar atual. $\mathrm{O}$ último registro dessas rochas é identificado a $+1,5 \mathrm{~m}$ acima do nível do mar atual, datado entre 3130 - $2860 \mathrm{cal}$ anos A.P. Esse intervalo de tempo, marca o início do fenômeno da ressurgência costeira no litoral de Cabo Frio, Estado do Rio de Janeiro.

\section{Referências Bibliográficas}

ALEXANDERSSON, T. Mediterranean beachrock cementation: marine precipitation of Mg-calcite. In: STANLEY, D. J. (Ed.). The Mediterranean Sea: A natural sedimentation laboratory. Stroudsburg, Dowden, Hutchinson \& Ross. p. 203-223. 1973.

ALMEIDA, F. F. M.; CARNEIRO, C. D. R.; MIZUSAKI, A. M. P. Correlação do magmatismo da margem continental brasileira com o das áreas emersas adjacentes. Revista Brasileira de Geociências. São Paulo, v. 26. n. 3. p. 125-138. 1996. (ISSN: 0375.7536).

ALMEIDA, F. F. M. O alinhamento magmático de Cabo Frio. In: Sociedade Brasileira de Geologia/Núcleos, São Paulo e Rio de Janeiro, $2^{\circ}$. Simpósio de Geologia do Sudeste, Atas, p. 423-428. 1991. 
AMBIENTAL, 2002. Plano de manejo da APA do Pau-Brasil/ Rio de Janeiro.

AMIEUX, P.; BERNIER, P.; DALONGEVILlE, R.; MEDWECKI, V. Cathodoluminescence of carbonate cemented Holocene beachrock from the Togo coastline (West Africa): an approach to early diagenesis. Sedimentary Geology. v. 65. n. 3-4. p. 261-272. 1989. (DÓI: 10.1016/0037-0738(89)90028/6).

BARBIÉRI, E. B. Ritmo climático e extração de sal em Cabo Frio, Revista Brasileira de Geografia, v. 37. n. 4. p. 23-109. 1975.

BARBOSA, D. S. Sedimentação Orgânica na Lagoa Brejo do Espinho (Cabo Frio, RJ): Composição e Implicações Paleoclimáticas. 2003. 90 f. Dissertação de Mestrado, Universidade Federal Fluminense. Niterói. 2003.

BECKER, B. An 11,000-year German oak and pine dendrochronology for radiocarbon calibration. Radiocarbon. v. 35. n. 1. p. 201-213. 1993. (DOI: 10.1017/S0033822200013898).

BEZERRA, F. H. R.; AMARAL, R. F.; LIMA-FILHO, F. P.; FERREIRA Jr., A. V.; SENA, E. S.; DINIZ, R. F. Beachrock fracturing in Brazil. Journal of Coastal Research. v. 42. p. 319-332. (Special Issue). 2005.

BEZERRA, F. H. R.; BARRETO, A. M. F.; SUGUIO, K. Holocene sea-level history on the Rio Grande do Norte State Coast, Brazil. Marine Geology. v. 196. n.1-2. p. 73-89. 2003. (DOI: 10.1016/S0025-3227(03)00044-6).

BIGARELLA, J. J. Reef sandstones from northeastern Brazil (A survey on sedimentary structures). Anais da Academia Brasileira de Ciências. v. 47. p. 395-409. 1975. (Suplemento).

BRANNER, J. C. The stone reefs of Brazil, their geological and geographical relations, with a chapter on the coral reefs. Museum of Comparative Zoology, Harvard College, Cambridge, Geological Series 7. 1904.

CALDAS, L. H. O.; STATTEGGER, K.; VITAL, H. Holocene sea level history: Evidence from coastal sediments of the Northern Rio Grande do Norte Coast, NE Brazil. Marine Geology. v. 228. p. 39-53. 2006. (ISSN 0025-3227).

CAstro, J. W. A.; SUGUiO, K.; SEOANE, J. C. S.; CUNHA, A. M.; DIAS, F. F. 2014. Sea-level fluctuations and coastal evolution in the State of Rio de Janeiro, Southeastern Brazil. Anais da Academia Brasileira de Ciências. v. 86. n. 2. p. 671-683. 2014. (DOI: 10.1590/0001-3765201420140007).

CAstro, J. W. A.; SENRA, M. C. E.; RAMOS, R. R. C. Coquinas da paleolaguna da Reserva Tauá - Pântano da
Malhada, Cabo Frio, RJ. In: Winge, M.; Schobbenhaus, C.; Souza C. R. G.; Berbet-Born, M; Queiroz, E. T.; Campos, D. A. (Eds). Sítios Geológicos e Paleontológicos do Brasil, vol. II, SIGEP, Brasília, Brasil, p. 269-276. 2009.

CASTRO, J. W. A.; SUGUIO, K.; CUNHA, A. M.; GUEDES, E.; TÂMEGA, F. T. S.; RODRIGUEZ, R. R. Rochas de Praia (Beachrocks) da Ilha do Cabo Frio, Arraial do Cabo: Registro Geológico Ímpar da Transição Pleistoceno - Holocenono Estado do Rio de Janeiro. Anuário do Instituto de Geociências Universidade Federal do Rio de Janeiro. v. 35. n.1. p. 236-241. 2012.

CHAVES, N. S.; SIAL, A. N. Mixed oceanic and freshwater depositional conditions for beachrocks of Northeast Brazil: Evidence from carbon and oxygen isotopes. International Geology Review. v. 40. p. 748-754. 1998.

(DOI: 10.1080/00206819809465235).

CHIVAS, A.; CHIPPELL, J.; POLACH, H.; PILLANS, B.; FLOOD, P. Radiocarbon evidence for the timing and rate of island development, beachrock formation and phosphatization at Lady Elliot Island, Queensland, Australia. Marine Geology. v. 69. n. 3-4. p. 273-287. 1986.

COOPER, J. A. G. Beachrock formation in low latitudes: implications for coastal evolutionary models. Marine Geology. v. 98. n. 1.p. 145-154. 1991.

COORAY, P. G. A note on the occurrence of beachrock along the west coast of Ceylon. Journal of Sedimentary Petrology. v. 38. n. 2. p. 650-654. 1968.

CPRM - Serviço Geológico do Brasil; BRASÍLIA 2001. PROGRAMA LEVANTAMENTOS GEOLÓGICOS BÁSICOS DO BRASIL.

DALONGEVILLE, R.; SANLAVILLE, P. Essai de synthèse sur le beach-rock. In: Dalongeville, R.; Sanlaville, P. (Ed.). Le beach-rock. Paris, Travaux de la Maison de l'Orient 8. Group ement d'Interets cientifique. p. 41-50. 1984.

DANA, J. D. United States Exploring Expedition. During the years $1838,1839,1840,1841,1842$. Under the command of Charles Wilkes, U.S.N. Vol. X. Geology, C. Sherman, Philadelphia. 1849.

DARWIN, C. R. On a remarkable bar of sandstone off Pernambuco, on the coast of Brazil. Edimburgh and Dublin Philosophical Magazine. v. 19. p. 257-260. 1841.

DIAS, G. T. M.; SILVA, C. G. Geologia de depósitos arenosos costeiros emersos - exemplos ao longo do litoral fluminense. In: UFF, Simpósio sobre Restingas Brasileiras, 1, Anais, p. 
47-60. 1984.

ELETROBRAS. Diretoria de Planejamento e Engenharia MEMORIA técnica de usinas hidrelétricas: roteiro básico. Rio de Janeiro: ELETROBRAS. 123p. 1988.

ERGINAL, A. E.; KIYAK, N. G.; BOZCU, M.; ERTEK, A.; GÜNGÜNES, H.; SUNGUR, A.; TÜRKER, G. On the origin and age of the Ariburnu Beachrock, Gelibolu Peninsula, Turkey. Turkish Journal of Earth Sciences. v. 17. n. 4. p. 803-819. 2008.

ERTEK, T. A.; ERGINAL, A. E.; BOZCU, M.; TÜRKER, G. The formation of beachrock on the North Cyprus Coast. Journal of Black Sea / Mediterranean Environment, Turkey. v. 14. n. 2. p. 117-128. 2008.

FERREIRA JUNIOR, A. V. 2005. Mapeamento da zona costeira protegida por arenitos de praia (beachrocks) em Nísia Floresta - RN. 2005. 105 f. Dissertação (Mestrado em Geodinâmica e Geofísica). Universidade Federal de Rio Grande do Norte - PPGG. Natal - RN. 2005.

FLEXOR, J. M.; MARTIN, L.; SUGUIO. K.; DOMINGUES, J. M. L. Gênese dos cordões litorâneos da parte central da Costa Brasileira. In: Lacerda, L.D., Araújo, D.S.D., Cerqueira, R. Turcq, B. (Orgs), Restingas: Origem, Estrutura, Processos. CEUFF, Universidade Federal Fluminense, Niterói, p. 35-46. 1984.

FONSECA, R. B. M. Evolução batimétrica e sedimentológica do banco de areia "sandbar" da Enseada dos Anjos, Arraial do Cabo, Rio de Janeiro. 92 f. 2012. Dissertação de Mestrado. Universidade Federal do Rio de Janeiro, Rio de Janeiro. 2012.

GINSBURG R. N. Beachrock in South Florida. Journal of Sedimentary Petrology. v. 23. n. 2. p. 85-92. 1953.

GOUDIE, A. A note on Mediterranean beachrock: its history. Atoll Research Bulletin. v. 126. p. 11-14. 1969.

GUERRA, N. C.; KIANG, C. H.; SIAL, A. N. Carbonate cements in contemporaneous beachrocks, Jaguaribe beach, Itamaracá island, Northeastern Brazil: petrographic, geochemical and isotopic aspects. NEG-LABISE, Departamento de Geologia, UFPE, Recife, PE, Brasil. Anais da Academia Brasileira de Ciências. Rio de Janeiro, v. 77, n. 2, p. 343-353, 2005.

GUILCHER, A. Le "beach-rock" ou grès de plage. Annales de Ge'ographie. v. 376. p. 113-125. 1961.

GYLLENCREUTZ, R.; MAHIQUES, M. M.; ALVES, D. V. P.; WAINER, I. K. C. Mid- to late-Holocene paleoceanographic changes on the southeastern Brazilian shelf based on grain size records. The Holocene, v. 20. n. 6. p. 863-875. 2010.
(DOI: 10.1177/0959683610365936).

HANOR, J. S. Precipitation of beachrock cements: mixing of marine and meteoric waters vs. CO2 degassing. Journal of Sedimentary Petrology. v. 48. n. 2. p. 489-501. 1978.

HOLAIL, H.; RASHED, M. Stable isotopic composition of carbonate-cemented recent beachrock along the Mediterranean and Red Sea Coasts of Egypt. Marine Geology. v. 106. n. 1. p. 141-148. 1992. (DOI: 10.1016/0025-3227(92)90059-Q).

HOPLEY, D. Beachrock as a sea-level indicator. In: Van de Plassche, O. (ed.). Sea-level research: a manual for collection and evaluation of data. Norwich, Geo Books. p. 157-173. 1986.

KHALAF, F.I. 1988. Quaternary calcareous hard rocks and the associated sediments in the intertidal and offshore zones of Kuwait. Marine Geology. v. 80. n. 1-2. p. 1-27. (DOI:10.1016/0025-3227(88)90069-2).

KINDLER, P.; BAIN, R. J. Submerged Upper Holocene on San Salvador Island, Bahamas: Implications for recent sea-level history. Geologisch Rundschau. v. 82. p. 241-427. 1993.

LASLANDES, B.; SYLVESTRE, F.; SIFEDDINE, A.; TURCQ, B.; ALBUQUERQUE, A. L. S.; ABRÃO, J. Enregistrement de la variabilité hydro-climatique au cours des 6500 dernières années sur lê litoral de Cabo Frio (Rio de Janeiro, Brésil). Comptes Rendus Geoscience. v. 338. n. 10. p. 667-675. 2006.

MABESOONE, J. M. Origin and age of the sandstone reefs of Pernambuco (Northeastern Brazil). Journal of Sedimentary Petrology. v. 34. p. 715-726. 1964.

MACEDO, A. C. M. Testemunhos geológicos da evolução do litoral no Estado da Guanabara. Anais da Academia Brasileira de Ciências. v. 43. n. 3-4 p. 832. 1963.

MALTA, J. V.; CASTRO, J. W. A.; OLIVEIRA, C. A.; REIS, C. C. Os Beachrocks são ou não são Indicadores Precisos de Variações do Nível Relativo do Mar? In: XV Congresso da Associação Brasileira de Estudos do Quaternário - ABEQUA \& II Encontro Brasileiro do Tecnógeno, Imbé/RS, Anais, 2, pp. 181. 2015.

MANSUR, K. L.; RAMOS, R. R. C.; GODOY, J. M. O.; NASCIMENTO, V. M. R. Beachrock de Jaconé, Maricá e Saquarema - RJ: importância para a história da ciência e para o conhecimento geológico. Revista Brasileira de Geociências. v. 41. n. 2. p. 290-303. 2011.

MARTIN, L.; SUGUIO, K.; FLEXOR, J. M.; AZEVEDO, A. E. G. Geologia do quaternário costeiro do litoral norte do Rio de Janeiro e do Espírito Santo. Belo Horizonte: CPRM. 
1997. 112 p.: il, mapas.

MARTIN, L.; FLEXOR, J. M.; SUGUIO, K. 1984. Enregistrement des périodes de fortes et faibles énergies à l'embouchure d'un fleuve. Le cas du Paraíba do Sul (Brésil). Implications paléoclimatiques. Comptes Rendus de l'Académie des Sciences II A. v. 299. p. 661-664.

MEYERS, J. H. Marine vadose beachrock cementation by cryptocrystalline magnesian calcite - Maui, Hawaii. Journal of Sedimentary Petrology. v. 57. p. 558-570. 1987.

MILLIMAN, J. D. Marine Carbonates. Springer-Verlag, Berlin, 1974. $375 \mathrm{p}$.

MORAIS, J. O. Contribuição ao estudo dos "Beachrocks" do nordeste do Brasil. Laboratório de Ciências do Mar da Universidade Federal do Ceará. Trabalhos Oceanográficos - Universidade Federal de Pernambuco. v. 9. p. 79-94. 1967.

MORESBY, R. M. Extracts from Commander Moresbys' report on the northern atolls of the Maldives. Journal of the Royal Geography Society of London. v. 5. p. 398-404. 1835.

MOTOKI, A.; SICHEL, S. E. Hydraulic fracturing as possible mechanism of dyke-sill transition and horizontal discordant intrusion: an example of Arraial do Cabo area, State of Rio de Janeiro, Brazil. Geofísica Internacional, México. v. 47. n. 1. p. 13-25. 2008.

MUEHE, D. C. E. H. Evidências de recuo dos cordões litorâneos em direção ao continente no litoral do Rio de Janeiro. In: Lacerda, L. D.; Araújo, D. S. D.; Cerqueira, R.; Turcq, B. (ed.), Restingas: origem, estruturas e processos. CEUFF - Universidade Federal Fluminense, 75-80. 1984.

MUEHE, D. C. E. H.; IGNARRA, S. O Arenito de Praia de Itaipuaçu e sua influência no fluxo de sedimentos. In: Lamego, A. R. (ed.). Anais do I Simpósio de Geologia Regional RJ-ES. Sociedade Brasileira de Geologia. Rio de Janeiro. p. 57-62. 1987.

NEUMEIER, U. Experimental modelling of beachrock cementation under microbial influence. Sedimentary Geology. v. 126. p. 35-46. 1999. (DOI: 10.1016/S0037-0738(99)00030-5).

NIMER, E. Climatologia do Brasil. Instituto Brasileiro de Geografia e Estatística, Departamento de Recursos Naturais e Ambientais. Rio de Janeiro, RJ, Brazil. 1989. 422 p.

RAMSAY, P. J. 9000 years of sea-level change along the Southern African coastline. Quatern Int. v. 31. p. 71-75. 1995.

RONCARATI, H.; NEVES, L. E. Estudo preliminar dos sedimentos recentes superficiais da Baixada de Jacarepaguá: Município do Rio de Janeiro (Projeto Jacarepaguá). Rio de
Janeiro: CENPES/ Petrobrás, 1976. 89 p.

RUSSELL, R. J. Caribbean beach rock observations. Zeitschrift für Geomorphologie. v. 3. p. 227-236. 1959.

RUSSELL, R.J. Origin of beach rock. Zeitschrift für Geomorphologie. v. 6. p. 1-16. 1962.

SCHMITT, R. S. A Orogenia Búzios - um evento tectonometamórfico cambro-ordoviciano caracterizado no Domínio Tectônico de Cabo Frio, Faixa Ribeira, sudeste do Brasil. 2001. 273 f. Tese de doutoramento. Instituto de Geociências da Universidade Federal do Rio de Janeiro, Rio de Janeiro. 2001.

SCHOLTEN, J. J. Beach rock: a literature study with special reference to the recent literature. Zentralblatt für Mineralogie und Paläontologie, v. 1. n. 9-10. p. 655-672. 1971.

SICHEL, S. E.; SCHIMIDDT, R. S.; ARAÚJO, A. L. N.; SKEREPNEK C.; CANABARRO, D.; ALVES, E. L.; GUERRA, J. V.; STANTON, N. Guia de Excursão de Campo à Ilha do Cabo Frio. III Simpósio de Vulcanismo e Ambientes Associados, Cabo Frio, RJ - Junho de 2005 - 22p. 2005

SILVA, A. L. C.; SILVA, M. A. M.; SOUZA, R. S.; PINTO, M. L.V. The role of beachrocks on the evolution of the Holocene barrier systems in Rio de Janeiro, southeastern Brazil. In: Green, A. N.; Cooper, J. A. G. (eds.). Proceedings 13th International Coastal Symposium (Durban, South Africa). Journal of Coastal Research, Special Issue No. 70, p. 170-175. 2014. (ISSN 0749-0208.).

SILVA, A. C.; CASTRO, J. W. A.; ALMEIDA, C. N.; DIAS, F. F. Caracterização de minerais pesados na plataforma continental interna de Cabo Frio, Rio de Janeiro, Brasil. Revista do Departamento de Hidrologia e Navegação. v. 68. p. 79-87. 2011.

SILVA, D. M. A. F.; SILVA, M. G. A. J. Para Entender Tempo e Clima. In: Cavalcanti, I. D. A.; Ferreira, N. J.; Da Silva, M. G. A. J.; Silva Dias, M.A.F. (Orgs.). Tempo e Clima no Brasil. São Paulo: Ofic. Textos. 2009. p. 15-21.

S POTORNO-OLIVEIRA, P.; TÂMEGA, F. T. S.; OlIVEIRA, C. A.; CASTRO, J. W. A.; COUTINHO, R.; IRYUC, Y.; BASSI, D. Effects of Holocene sea level changes on subtidal palaeoecosystems, southeastern Brazil. Marine Geology, v. 381. n. 1. p. 17-28. 2016. (DOI: 10.1016/j. margeo.2016.08.007).

STODDART, D. R.; CANN, J. R. Nature and origin of beach rock. Journal of Sedimentary Petrology. v. 35. p. 243-273. 1965.

SUGUIO, K. Geologia Sedimentar, 1a. ed., Editora Edgard Blücher, São Paulo, 2003a. 324 p.

SUGUIO, K. Tópicos de geociências para o desenvolvimento 
sustentável: as regiões litorâneas. Boletim de Geologia da Universidade de São Paulo: Série Didática. v. 2. n. 1. p. 1-40. $2003 b$.

SUGUIO, K.; TESSLER, M, G. 1984. Planícies de cordões litorâneos quaternários do Brasil: origem e nomenclatura. In: Lacerda, L. D.; Araújo, D. S. D.; Cerqueira, R.; Turcq, B. (Eds). Restingas: origem, estrutura, processos, CEUFF, Niterói, p. 15-25.

SUGUIO, K.; MARTIN, L. Classificação de costas e evolução geológica das planícies litorâneas quaternárias do sudeste e sul do Brasil. In: ACIESP (Orgs). Simpósio de Ecossistemas da Costa Sul e Sudeste Brasileira, Anais v. 1. p. 1-28. 1987.

SUGUIO, K. Geologia do Quaternário e mudanças ambientais. Passado + Presente - Futuro. Paulo's Editora, São Paulo. 1999. $236 \mathrm{p}$.

TERRA, G. J. S. Fácies, Modelo Deposicional e Diagênese da Sequência Carbonática Albo-Cenomaniana (Formação Ponta do Mel) da Bacia Potiguar. Tese de Mestrado. 2010. 153 f. Universidade Federal do Rio de Janeiro. 2010.

THOMAS, P. J. Luminescence dating of beachrock in the southeast coast of India. Potential for Holocene Shoreline Reconstruction. Journal of Coastal Research. v. 25. n. 1. p. 1-7. 2009.

TIETZ, G.; MÚLLER, G. High-magnesian calcite and aragonite cementation in recent beachrocks, Fuerteventura, Canary Islands, Spain. In: Bricker, O. P. (ed.). Carbonate Cement. Tite Johns Hopkins University, Baltimore and Londres. 1971. p. 4-8.

TURCQ, B. Origin and evolution of the quaternary coastal plain between Guaratiba and Cabo Frio, State of Rio de Janeiro, Brazil. In: Knoppers, B. et al. (org.). Environmental Geochemistry of Coastal Lagoon Systems, Rio de Janeiro, Brazil, Universidade Federal Fluminense, Série Geoquímica Ambiental. v. 6. p. 25-46. 1999.
VAN ANDEL, T. H.; LABOREL, J. Recent high relative sea level stand near Recife, Brazil. Reprinted from Science, v. 3632. p. 580-581. 1964.

VIEIRA, M. M. Aspectos sedimentológicos e petrológicos dos beachrocks do Estado do Rio Grande do Norte. Tese de Doutorado. 2005. 243 f. Universidade Federal do Rio Grande do Sul, Porto Alegre. 2005.

VIEIRA, M. M.; De ROS, L. F.; BEZERRA, F. H. R. Lithofaciology and Palaeoenvironmental Analysis of Holocene Beachrocks in Northeastern Brazil. Journal of Coastal Research. West Palm. v. 23. n. 6. p. 1535-1548. 2007.

VIEIRA, M. M.; De ROS, L. F. Cementation patterns and genetic implications of Holocene beachrocks from northeastern Brazil. Sedimentary Geology. v. 192. p. 207-230. 2006.

VILLWOCK, J. A. A costa brasileira: geologia e evolução. Notas Técnicas. Universidade Federal Rio Grande do Sul. v. 7. p. 38-49. 1994. (ISSN 0104-4303)

VON BUCH, L. Physikalische Beschreibung der Canarischen Inseln. Hofdruckerei der Königlichen Akademie, Berlin. 1825 .

WEBB, G. E.; JELL, J. S.; BAKER, J. C. Cryptic intertidal microbialites in beachrock, Heron Island, Great Barrier Reef: implications for the origin of microcrystalline beachrock cement. Sedimentary Geology. v. 126. p. 317-334. 1999. (DOI:10.1016/S0037-0738(99)00047-0).

ZIMBRES, E. K.; KAWASHITA, K.; VAN SCHMUS, W. R. Evidências de um núcleo transamazônico na região de Cabo Frio, RJ e sua conexão com o Cráton de Angola. In: XXXVI CONGRESSO BRASILEIRO DE GEOLOGIA, Natal, RN, SBG, Anais, v. 6. p. 2735-2743. 1990. 